\title{
TITLE:
}

\section{Use of helium double ionization for autocorrelation of an xuv pulse}

AUTHOR(S):

Nakajima, T; Nikolopoulos, LAA

CITATION:

Nakajima, $T$...[et al]. Use of helium double ionization for

autocorrelation of an xuv pulse. PHYSICAL REVIEW A 2002, 66(4):

041402.

ISSUE DATE:

2002-10

URL:

http://hdl.handle.net/2433/50415

RIGHT:

Copyright 2002 American Physical Society 
PHYSICAL REVIEW A 66, 041402(R) (2002)

\title{
Use of helium double ionization for autocorrelation of an xuv pulse
}

\author{
Takashi Nakajima ${ }^{1, *}$ and L. A. A. Nikolopoulos ${ }^{2}$ \\ ${ }^{1}$ Institute of Advanced Energy, Kyoto University, Gokasho, Uji, Kyoto 611-0011, Japan \\ ${ }^{2}$ Institute of Electronic Structure and Laser, F.O.R.TH, P.O. Box 1527, Heraklion 711 10, Crete, Greece
}

(Received 7 August 2002; published 30 October 2002)

\begin{abstract}
We investigate a simple scheme for autocorrelation measurement of an xuv pulse. It is based on double ionization of He. We have found that, in a certain photon energy range, the detection of doubly charged positive ions instead of energy-resolved photoelectrons is sufficient for autocorrelation, which greatly simplifies the detection system for practical use.
\end{abstract}

DOI: 10.1103/PhysRevA.66.041402

PACS number(s): 42.50.Hz, 42.65.Ky, 32.80.Rm

In recent years, efforts have been and are still being made to develop ultrashort-pulse lasers in the vacuum ultraviolet (vuv) extreme ultraviolet (xuv) region. So far there are two distinct approaches toward that direction.

The first one is the high harmonic generation (HHG) from a femtosecond Ti:sapphire laser, where the generated xuv pulse is necessarily accompanied by a near-infrared fundamental pulse with sufficient intensity. So far the autocorrelation measurement was successful only up to the 9th harmonic [1] due to the insufficient intensity to induce nonlinear phenomena. In order to circumvent this problem, various schemes, which are essentially variants of cross correlation [2-11], have been invented to measure the pulse duration of an HHG-xuv pulse. Briefly, due to the presence of an intense fundamental pulse, the intensity and shift of the sidebands in the photoelectron signal produced by the HHG-xuv pulse depend on the time delay between the HHG-xuv and fundamental pulses. Since the delay is variable by using an optical delay line or a dispersive element, the sideband signal can be utilized for cross correlation. It should be noted that an HHG-xuv pulse can have a subfemtosecond pulse duration if the high harmonics are superposed phase-coherent, as demonstrated in Refs. [4-6,12,13]. A usual cross-correlation scheme does not work for such a short pulse, since the time resolution is limited by the fundamental pulse whose duration extends from a few femtoseconds $[4,5]$ to tens of femtoseconds $[7,6]$. In order to obtain higher (subfemtosecond) temporal resolution, one must employ a phase-dependent cross correlation. Note that all of these techniques are possible because of the simultaneous presence of the fundamental and xuv pulses.

The second one is a free-electron laser (FEL), which is, for example, represented by the TESLA Test Facility (TTF) at the Deutsches Elektronen-Synchrotron (DESY). They have successfully generated intense vuv pulses in the wavelength of 95-105 nm with an energy of 30-100 $\mu \mathrm{J} /$ pulse and an estimated pulse duration of 30-100 fs. The outcoming beam diameter is reported to be $250 \mu \mathrm{m}$, resulting in an intensity of $>6 \times 10^{12} \mathrm{~W} / \mathrm{cm}^{2}[14-16]$ without focus. If the upgrading of the FEL goes well, an intense FEL-xuv pulse will be available in the next few years. A detailed characterization of the FEL-xuv pulse, however, has not yet been

\footnotetext{
*Email address: t-nakajima@iae.kyoto-u.ac.jp
}

performed: The pulse duration was indirectly estimated from the bunching of electrons. The difference between the FELxuv pulse and the HHG-xuv pulse is that the former is not accompanied by a synchronized near-infrared pulse, which makes a cross correlation very difficult. The intensity of the FEL-xuv pulse, however, is sufficient to induce nonlinear phenomena. Obviously a direct pulse width measurement is desired for a femtosecond FEL-xuv pulse.

So far no theoretical analysis has been reported for autocorrelation of an xuv pulse. This is because there has been no light source available, until now, with sufficient intensity for autocorrelation in the xuv regime. As described above, however, the time is about to come for the intense FEL-xuv pulse. We note further that a significant improvement in intensity for the HHG-xuv pulse has been recently reported [17]: Using a loose-focus geometry, they have obtained a few $\mu \mathrm{J}$ energy for each 11-15th HHG pulse, and $0.3 \mu \mathrm{J}$ energy for the 27th HHG generated from a 35 fs Ti:sapphire laser. By focusing into the $5 \mu \mathrm{m}$ spot size and assuming that the pulse duration of the 27th HHG is about 35 fs, the intensity can be $>4 \times 10^{14} \mathrm{~W} / \mathrm{cm}^{2}$, which is much more than sufficient to induce two-photon processes. It is these considerations that lead us to the quantitative investigation of an autocorrelation scheme for the ultrashort xuv pulse, which could serve as a benchmark for future measurements.

In this paper, we analyze an autocorrelation scheme for an xuv pulse, using $\mathrm{He}$ gas as a nonlinear medium, through the detection of doubly charged $\mathrm{He}^{2+}$ ions. In particular, as depicted in Fig. 1, we focus on the photon energy range of 40-54.4 eV, where two and three photons are required for direct and sequential double ionization, respectively [18]. Since direct double ionization is a lower-order process, it might dominate for a certain range of intensities and pulse durations, as we will show in this paper. This means that the two-photon double ionization signal can be used for the autocorrelation measurement. One may ask why we do not look at the two-photon above-threshold ionization (ATI). Of course, that can be used for autocorrelation as well. The advantage of using the double ionization instead of ATI is that in the former it is doubly charged ions that are used for autocorrelation, while in the latter it is energy-resolved photoelectrons. Needless to say, the charge-selected detection of positive ions is technically much easier than the energyresolved detection of photoelectrons. Before moving onto the description of the scheme, we note that our analysis is based 


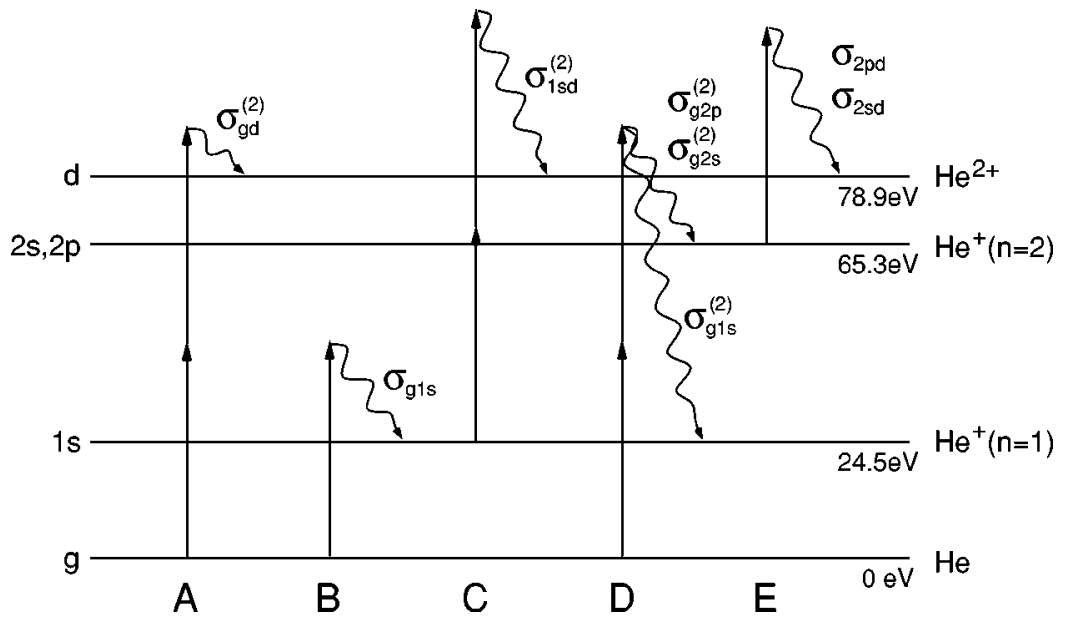

FIG. 1. Level scheme considered in this paper.

on the rate equations together with a detailed atomic structure calculation armed with a multichannel $B$-spline method, which can fully take into account the configuration interaction among bound, single-continuum, and double-continuum states $[19,20]$.

The scheme we investigate is depicted in Fig. 1. Helium atoms in the ground state interact with an xuv pulse. States $g$, $1 s, 2 s, 2 p$, and $d$, respectively, describe the ground-state He $1 s^{2}$, singly ionized states $\mathrm{He}^{+} 1 s, 2 s$, and $2 p$, and doubly ionized state $\mathrm{He}^{2+}$. In the photon energy range we have chosen, direct double ionization requires two photons (path $A)$. Physically direct double ionization means that the two electrons are simultaneously ejected with kinetic energies such that their sum is equal to the excess energy above the double-ionization threshold. Sequential double ionization requires at least three photons (path $B+$ path $C$ and path $D$ + path $E$, etc.). It should be noted that the ionic states other than $n=1$ and $n=2$ have been neglected in our model, the justification for which will be given later in this paper.

If we are to look into the photoelectron energy spectrum with or without resonance, a set of amplitude equations or density-matrix equations has to be used for the analysis $[18,20]$. However, if the photon energy is chosen such that there is no resonance involved, and the population of each charge state, $R_{g}$ (neutral), $R_{1 s}+R_{2 s}+R_{2 p}$ (singly ionized), and $R_{d}$ (doubly ionized), is of interest, a set of rate equations is sufficient to describe the system:

$$
\begin{gathered}
\dot{R}_{g}=-\left[\left(\sigma_{g d}^{(2)}+\sum_{k=1 s, 2 s, 2 p} \sigma_{g k}^{(2)}\right) I_{F}^{2}+\sigma_{g 1 s} I_{F}\right] R_{g}, \\
\dot{R}_{1 s}=\left(\sigma_{g 1 s}^{(2)} I_{F}^{2}+\sigma_{g 1 s} I_{F}\right) R_{g}-\sigma_{1 s d}^{(2)} I_{F}^{2} R_{1 s}, \\
\dot{R}_{k}=\sigma_{g k}^{(2)} I_{F}^{2} R_{g}-\sigma_{k d} I_{F} R_{k} \quad(k=2 s, 2 p), \\
\dot{R}_{d_{1}}=\sigma_{g d}^{(2)} I_{F}^{2} R_{g}, \\
\dot{R}_{d_{2}}=\sigma_{1 s d}^{(2)} I_{F}^{2} R_{1 s}+\sigma_{2 s d} I_{F} R_{2 s}+\sigma_{2 p d} I_{F} R_{2 p},
\end{gathered}
$$

where $\sigma_{i j}^{(2)}$ 's and $\sigma_{i j}$ 's are, respectively, the two- and singlephoton ionization cross sections from state $i$ to $j . R_{d_{1}}$ and $R_{d_{2}}$ denote the double-ionization yields via the direct and sequential processes, respectively. $I_{F}$ (photons $/ \mathrm{cm}^{2} \mathrm{~s}$ ) represents a photon flux, and it is connected to the intensity $I$ $\left(\mathrm{W} / \mathrm{cm}^{2}\right)$ through $I_{F}=I / \hbar \omega$, where $\hbar \omega(\mathrm{J})$ is a photon energy.

For a square pulse with a duration $\tau$, it is trivial to solve these equations analytically, and under the weak excitation in that $R_{g} \sim 1$ at all time, we obtain $R_{d_{1}} \simeq \sigma_{g d}^{(2)} I_{F}^{2} \tau$ for direct two-photon double ionization and $R_{d_{2}} \simeq 0.5\left(\sigma_{g 1 s} \sigma_{1 s d}^{(2)}\right.$ $\left.+\sigma_{g 2 s}^{(2)} \sigma_{2 s d}+\sigma_{g 2 p}^{(2)} \sigma_{2 p d}\right) I_{F}^{3} \tau^{2}$ for sequential double ionization, where the first and the rest of the terms represent sequential $(1+2)$ - and $(2+1)$-photon double ionization, respectively.

For the present study, we specifically assume the photon energy of $45 \mathrm{eV}$. Using a multichannel $B$-spline code developed in recent years $[19,20]$, we obtain the cross sections as $\sigma_{g d}^{(2)}=8.1 \times 10^{-52} \mathrm{~cm}^{4} \mathrm{~s}, \quad \sigma_{g 1 s}^{(2)}=1.0 \times 10^{-52} \mathrm{~cm}^{4} \mathrm{~s}, \quad \sigma_{g 2 s}^{(2)}$ $=2.3 \times 10^{-51} \mathrm{~cm}^{4} \mathrm{~s}, \quad \sigma_{g 2 p}^{(2)}=3.8 \times 10^{-51} \mathrm{~cm}^{4} \mathrm{~s}, \quad \sigma_{g 1 s}=2.4$ $\times 10^{-18} \mathrm{~cm}^{2}, \quad \sigma_{1 s d}^{(2)}=1.0 \times 10^{-53} \mathrm{~cm}^{4} \mathrm{~s}, \quad \sigma_{2 s d}=2.4$ $\times 10^{-19} \mathrm{~cm}^{2}$, and $\sigma_{2 p d}=9.0 \times 10^{-20} \mathrm{~cm}^{2}$. In our scheme the accuracy of the double-ionization cross section $\sigma_{g d}^{(2)}$ is of particular importance. It should be noted that the difference between our cross section, obtained from a multichannel $B$-spline method, and the one obtained by using a product of Coulomb wave functions for the double continuum together with a truncated summation for intermediate states for $1 \operatorname{snp}(n \leqslant 10)$ [18], is only one order of magnitude, which is rather small, considering the crude approximations employed in Ref. [18]. Our number compares quite well with those reported in Refs. [21,22] within only $30 \%$ difference. Therefore, we consider that our $\sigma_{g d}^{(2)}$ must be within a factor of 2 accuracy from the real value.

If we assume an intensity of $I=2 \times 10^{12} \mathrm{~W} / \mathrm{cm}^{2}$ and a square pulse of $\tau=50$ fs duration, we obtain, from the analytical solutions given above, that $R_{d_{1}}=3.1 \times 10^{-6}$ and $R_{d_{2}}$ $=2.4 \times 10^{-8}$, revealing that the direct double-ionization yield is more than two orders of magnitude larger than the sequential double-ionization yield [18], i.e., $R_{d_{1}}+R_{d_{2}}$ $\simeq R_{d_{1}}$. This is the reason why the total double-ionization signal $\mathrm{He}^{2+}$ can be used for autocorrelation. It is worth noting that the $(2+1)$-photon double-ionization yield is about 

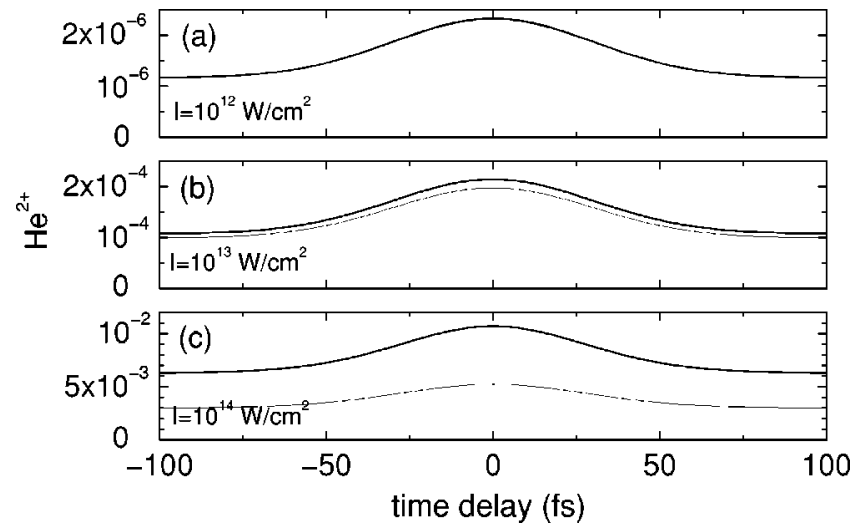

FIG. 2. Variation of the yield of doubly charged ions $\mathrm{He}^{2+}$, produced by the direct and the sequential processes, as a function of time delay between two identical pulses. Pulse duration is $50 \mathrm{fs}$ (FWHM) with a Gaussian profile. (a) $I=10^{12} \mathrm{~W} / \mathrm{cm}^{2}$, (b) $I$ $=10^{13} \mathrm{~W} / \mathrm{cm}^{2}$, and (c) $I=10^{14} \mathrm{~W} / \mathrm{cm}^{2}$. In each graph, total yield of the direct and the sequential processes (solid line) and the yield by the direct process only (thin solid line) are plotted.

40 times larger than that of the $(1+2)$-photon double ionization. Regarding path $D$, we have found that the contribution of the branching into the ionic states with higher $n$ $(\geqslant 3)$ is about $13 \%$ with respect to the total two-photon single-ionization rates. Knowing this and the single-photon ionization rate from those ionic states, we estimate that the contribution of the ionic states neglected in path $D$ of Fig. 1 to sequential double ionization is less than $5 \%$. Therefore, we can safely neglect those ionic states in path $D$.

Now, to be more realistic, we numerically solve the rate equations for a time-dependent pulse. In Fig. 2, we show a variation of the yield of doubly charged ions, $\mathrm{He}^{2+}: R_{d_{1}}$ for the direct process only and $R_{d_{1}}+R_{d_{2}}$ for the total (direct plus sequential) process, as a function of pulse delay at three different intensities $I=10^{12}, 10^{13}$, and $10^{14} \mathrm{~W} / \mathrm{cm}^{2}$ for each pulse. The pulse duration has been chosen to be $50 \mathrm{fs}$ [full width at half maximum (FWHM)] with a Gaussian temporal profile. It is perhaps useful to estimate how many doubly charged ions we can get per pulse. If we assume the intensity and the pulse duration to be $2 \times 10^{12} \mathrm{~W} / \mathrm{cm}^{2}, 50 \mathrm{fs}$ (Gaussian), the $\mathrm{He}^{2+}$ yield is $\sim 10^{-6}$, which means that the number of $\mathrm{He}^{2+}$ ions is about 20/pulse under the He gas pressure of 5 Torr and $125 \mu \mathrm{m}^{3}$ interaction volume.

Next we examine the variation of the autocorrelation width, determined from the FWHM presented in Fig. 2, as a function of intensity of each pulse for four different pulse durations, $100 \mathrm{fs}, 50 \mathrm{fs}, 30 \mathrm{fs}$, and $10 \mathrm{fs}$. In order to see the contribution of sequential double ionization, we plot three curves in Fig. 3 for each pulse duration, corresponding to the autocorrelation widths determined from the direct signal only, sequential signal only, and the total signal. We can see that the difference is practically negligible for the pulse durations shorter than 50 fs. At the intensity of $>2$ $\times 10^{13} \mathrm{~W} / \mathrm{cm}^{2}$ for the $100 \mathrm{fs}$ pulse, sequential double ionization takes place during the long pulse duration, and therefore the FWHM is different compared with that at lower intensities. To see the sensitivity of the determined autocorrelation

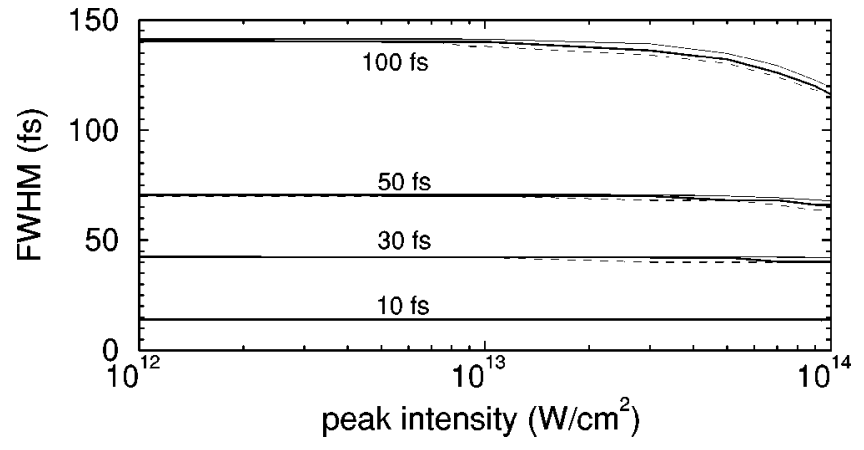

FIG. 3. Autocorrelation width (FWHM) determined from the numerical solution of Eqs. (1)-(5) as a function of intensity for four different pulse duration, $100 \mathrm{fs}, 50 \mathrm{fs}, 30 \mathrm{fs}$, and $10 \mathrm{fs}$ with a Gaussian profile. FWHM determined from the direct doubleionization yield (thin solid line), sequential double-ionization yield (thin dashed line), and the total double-ionization yield (solid line).

width on the double-ionization cross section $\sigma_{g d}^{(2)}$ whose accuracy may not be as good as other parameters, we have set $\sigma_{g d}^{(2)}$ to be zero as an extreme test, and repeated the calculation, finding a negligible difference (not shown). This means that, although sequential $(1+2)$ - and $(2+1)$-photon double ionization has third-order nonlinearity for a single pulse, its nonlinearity is very close to the second order for timedelayed pulse pair. Similar calculations have been performed for a sech ${ }^{2}$ pulse. Again we have found results (not shown here) very similar to those presented in Fig. 3. These results are quite encouraging in the context of using a total doubleionization signal for autocorrelation of an xuv pulse.

In the present work, we have specifically assumed the photon energy of $45 \mathrm{eV}$. It is meaningful to examine whether our scheme works at different photon energy for $40 \mathrm{eV}$ $<\hbar \omega<54.4 \mathrm{eV}$. Among the various ionization cross sections used here, $\sigma_{g d}^{(2)}, \sigma_{g 1 s}^{(2)}, \sigma_{g 2 s}^{(2)}, \sigma_{g 2 p}^{(2)}, \sigma_{g 1 s}, \sigma_{1 s d}^{(2)}, \sigma_{2 s d}$, and $\sigma_{2 p d}$, it is only $\sigma_{1 s d}^{(2)}$ that significantly changes its value at different photon energies simply because of the level structure of $\mathrm{He}^{+}$. Using a Green-function technique, we have calculated the variation of $\sigma_{1 s d}^{(2)}$. The result is shown in Fig. 4. It can be seen that the variation of its value is within one order of magnitude as long as the photon energy is not very close to resonance. Recalling that the Fourier width of the 50 fs pulse is $<0.08 \mathrm{eV}$ and is still $<1 \mathrm{eV}$ for a $10 \mathrm{fs}$ pulse,

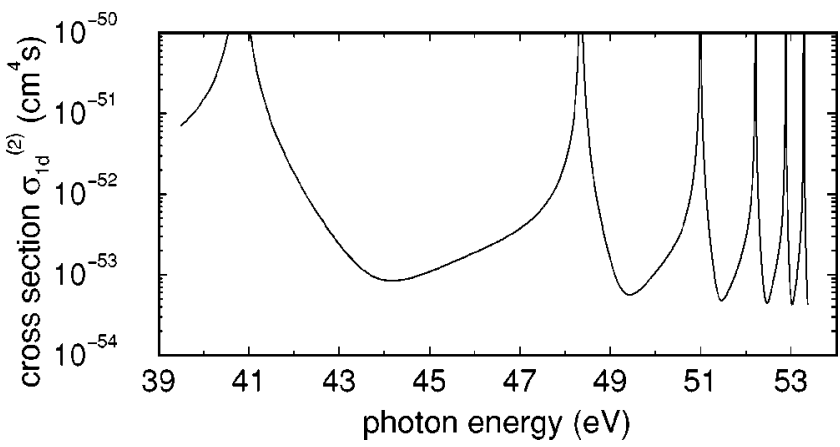

FIG. 4. Two-photon ionization cross section for $\mathrm{He}^{+}$to $\mathrm{He}^{2+}$ as a function of photon energy. 
this is not a serious problem. The variation of all other cross sections turned out to be within $30 \%$ difference from those at $45 \mathrm{eV}$ in the entire range of $40 \mathrm{eV}<\hbar \omega<54.4 \mathrm{eV}$, and does not affect our conclusion.

For a shorter pulse, say, $<5$ fs, the Fourier width may be so broad to cause near-resonance, leading to the undesired enhancement of sequential double ionization. For such a short pulse, a time-dependent analysis, for example using a set of amplitude equations, may be necessary to extract the pulse width from the experimentally obtained autocorrelation width.

One may wonder if our scheme can be extended for an xuv pulse with higher photon energy, $54.4<\hbar \omega<78.9 \mathrm{eV}$, where both direct and sequential double-ionization processes require two photons. In such a photon-energy range, sequential two-photon double ionization is dominant over direct two-photon double ionization, because it is typically such that $\sigma_{g d}^{(2)} \ll \sigma_{g n l} \sigma_{n l d}$ for He [18], where $n, l$ are the principal quantum number and the orbital angular momentum, respectively. One may still argue, however, that sequential twophoton double ionization may be used for autocorrelation. Although both direct/sequential double-ionization signals have second-order nonlinearity for a single pulse, it is not so for a time-delayed pulse pair: By solving a set of rate equations similar to Eqs. (1)-(5), we have found that the sequential double-ionization signal has no delay dependence, since the process does not require the simultaneous absorption of two photons. Therefore, our autocorrelation scheme does not work for $54.4<\hbar \omega<78.9 \mathrm{eV}$. Of course, if ionic species are used as a nonlinear medium for two-photon double ionization, one can always make a similar analysis at the expense of elaborate experimental setup to prepare ions prior to the autocorrelation measurement and smaller cross sections, which requires higher intensity for an xuv pulse. For such a big photon, there may be no other choice but to detect energy-resolved photoelectrons coming out of the twophoton ATI.

In summary, we have quantitatively examined a simple scheme for autocorrelation of an ultrashort xuv pulse. It is based on double ionization of He. Under the photon-energy range of $40 \mathrm{eV}<\hbar \omega<54.4 \mathrm{eV}$, doubly charged positive ions, $\mathrm{He}^{2+}$, can be used for the autocorrelation measurement, since direct two-photon double ionization is dominant over sequential $(1+2)$ - and $(2+1)$-photon double ionization. We have also found that, even if sequential $(1+2)$ - and $(2+1)$-photon double ionization is dominant over direct double ionization, the total double-ionization signal can be used for autocorrelation. We would like to emphasize once more that the detection of doubly charged positive ions is technically much easier than that of energy-resolved photoelectrons, which makes our scheme practical.

T.N. acknowledges useful discussions with Dr. K. Midorikawa. L.N. acknowledges the financial support of the Kyoto University Foundation for his stay at Kyoto University, where part of this work was carried out. The work by T.N. was supported by a Grant-in-Aid for Scientific Research from the Ministry of Education and Science of Japan.
[1] Y. Kobayashi et al., Opt. Lett. 23, 64 (1998).

[2] T.E. Glover et al., Phys. Rev. Lett. 76, 2468 (1996).

[3] J.M. Schins et al., J. Opt. Soc. Am. B 13, 197 (1997).

[4] M. Drescher et al., Science 291, 1923 (2001).

[5] M. Hentschel et al., Nature (London) 414, 509 (2001).

[6] P.M. Paul et al., Science 292, 1689 (2001).

[7] E.S. Toma et al., Phys. Rev. A 62, 061801(R) (2000).

[8] J. Itatani et al., Phys. Rev. Lett. 88, 173903 (2002).

[9] M. Kitzler et al., Phys. Rev. Lett. 88, 173904 (2002).

[10] J. Norin et al., Phys. Rev. Lett. 88, 193901 (2002).

[11] T. Sekikawa et al., Phys. Rev. Lett. 88, 193902 (2002).

[12] I.P. Christov et al., Phys. Rev. Lett. 78, 1251 (1997).

[13] N.A. Papadogiannis et al., Phys. Rev. Lett. 83, 4289 (1999).

[14] J. Andruszkow et al., Phys. Rev. Lett. 85, 3825 (2000).
[15] V. Ayvazyan et al., Phys. Rev. Lett. 88, 104802 (2002).

[16] V. Ayvazyan et al., Eur. Phys. J. D 20, 149 (2002).

[17] E. Takahashi et al., Phys. Rev. A 66, 021802(R) (2002).

[18] M.A. Kornberg and P. Lambropoulos, J. Phys. B 32, L603 (1999).

[19] L.A.A. Nikolopoulos and P. Lambropoulos, J. Phys. B 34, 545 (2001).

[20] L. A. A. Nikolopoulos, T. Nakajima, and P. Lambropoulos (unpublished).

[21] J. Colgan and M.S. Pindzola, Phys. Rev. Lett. 88, 173002 (2002); L. A. A. Nikolopoulos and P. Lambropoulos (unpublished).

[22] T. Mercouris, C. Haritos, and C. Nicolaides, J. Phys. B 34, 3789 (2001). 